\title{
Gerontopsychiatrie: vom Rand ins Zentrum
}

\author{
Geriatric Psychiatry: from the Margins to the Center
}

Autor

Institut
Hans Gutzmann

Abteilung für Psychiatrie und Psychotherapie, Krankenhaus Hedwigshöhe, Berlin

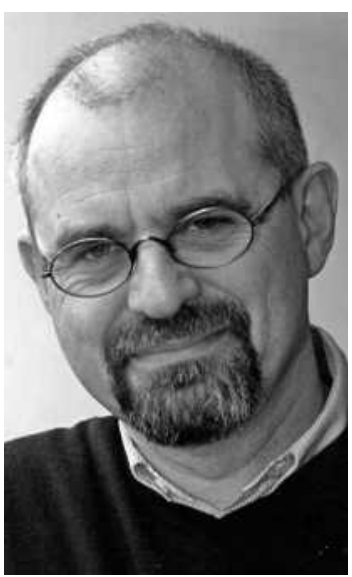

Prof. Dr. Hans Gutzmann
Bibliografie

DOI 10.1055/s-2007-970801

Psychiat Prax 2007; 34:

105-107 @ Georg Thieme

Verlag KG Stuttgart · New York .

ISSN 0303-4259

Korrespondenzadresse

Prof. Dr. Hans Gutzmann

Abteilung für Psychiatrie und

Psychotherapie, Krankenhaus

Hedwigshöhe

Höhensteig 1

12526 Berlin

h.gutzmann@alexius.de
Nach gut zwei Jahren widmet die Psychiatrische Praxis erneut ein Themenheft der Psychiatrie der alten Patienten. Dies kann wohl neben manchem anderen Zeichen als Hinweis darauf gelten, dass die Gerontopsychiatrie im fachlichen Spektrum, das die Psychiatrie abdeckt, immer deutlicher wahrgenommen wird. Bei der Psychiatrie des alten Menschen handelt es sich aber nicht um eine möglicherweise recht interessante, aber angesichts wichtigerer Herausforderungen doch wohl eher zu vernachlässigende Petitesse unseres Fachs. Es geht vielmehr um die Zukunft der Psychiatrie selbst. Altern wird in unseren Tagen zum ersten Mal zum Massenphänomen. Viele der heute unter 50-jährigen Kolleginnen und Kollegen werden erleben, was heute schon in Praxen in Brandenburg oder Mecklenburg-Vorpommern üblich ist: die Mehrheit der Patientinnen und Patienten ist älter als 65 Jahre. Dies wird auch in anderen Regionen der Republik psychiatrischer Alltag werden.

Spätestens zu diesem nicht mehr fernen Zeitpunkt wird sich die Argumentation über das Pro und Kontra einer separierten psychiatrischen Versorgung [1] umkehren. Spätestens dann dürften Stimmen immer lauter werden, die Schutzund Rückzugsräume für jüngere psychisch Kranke reklamieren, da man denen die gerontopsychiatrisch geprägte Umgebung integriert arbeitender Stationen nicht mehr zumuten könne. Die Zahl der psychisch kranken Älteren wird auch ohne den Einbezug der Demenzkranken in den nächsten 30 Jahren um 275\% zunehmen, weil die Lebenserwartung älterer psychisch Kranker steigen und die Mortalität jüngerer psychisch Kranker sinken wird.

Als zusätzliches Problem ist zu erwarten, dass die Zahlen der Leichtkranken durch Prophylaxe und intensiviertes Casefinding steigen und den Druck auf ambulante Strukturen erhöhen wird, während die Zahl der Schwerkranken mit ihrem stationären Behandlungsbedarf wegen der steigen- den Lebenserwartung auch dieser Gruppe überproportional zunehmen dürfte [2]. Vorsorge zu treffen für dieses demografische und epidemiologische Szenario heißt, sich darüber klar zu werden, dass wir in einem alternden Land alt werden, in dem immer noch weitgehend darüber Konsens besteht, dass dem Alter nur wenige gute Seiten abzugewinnen sind. Seine mediale Präsenz lässt es bestenfalls als amüsant bis lächerlich, schlimmstenfalls als bedrohlich dastehen. Positiv konnotierte Altersweisheit ist trotz interessanter Forschungsansätze [3] in der öffentlichen Wahrnehmung immer noch eher eine romantische Reminiszenz und kein Alltagsphänomen. Solange Altern im öffentlichen Bewusstsein vermeintlich immer nur die anderen betrifft, wird sich das Dilemma nicht lösen lassen.

Denjenigen unter den Leserinnen und Lesern, die sich gerontopsychiatrischen Fragen bisher eher aus der Distanz gewidmet und Hebephrenien eher dem Kernbestand psychiatrischer Erkrankungen zugerechnet haben als Demenzen, sei somit nahe gelegt, sich umzustellen: Babyboomer, tua res agitur! Hoffnung für die Zukunft gibt in diesem Zusammenhang ein aktueller epidemiologischer Befund [4], nach dem Erkrankungen, die in der eigenen Altersgruppe häufiger sind, auch mehr positive Aufmerksamkeit genießen. Gegenüber den alten Patienten hat die deutsche Psychiatrie eine Bringschuld. Im stationären Bereich hatte sie im Zeitraum zwischen 1994 und 1997 im Gegensatz zu allen anderen medizinischen Fächern eine Abnahme des Anteils älterer Patienten zu verzeichnen. Während im Bereich der psychiatrischen Krankenhäuser nur eine geringe Reduktion von 17,6 auf 16,7\% stattfand, war für die psychiatrischen Abteilungen ein drastisches Absinken von 14,6 auf 12,4\% zu beobachten [5]. Die gleichzeitig deutlich angestiegene Kapazität an Heimplätzen legte den Gedanken nahe, dass viele alte psychisch Kranke direkt von der Häuslichkeit ohne kompetente Intervention 
eines Facharztes oder einer Fachabteilung direkt ins Heim transferiert wurden [6]. Möglicherweise ging diese Entwicklung in den stationären Bereichen auch mit einer Vernachlässigung der Hochaltrigen und Verschiebung des diagnostischen Spektrums zulasten der organisch begründeten psychischen Erkrankungen einher, wie eine Studie der Bundesarbeitsgemeinschaft der Träger psychiatrischer Krankenhäuser [7] nahe legt.

Sehr ähnlich stellt sich das Problem in den USA dar, wo Ettner [8] bei der Analyse von 170000 Medicare-Patienten feststellte, dass die Aufnahme in einer psychiatrischen Fachabteilung desto unwahrscheinlich wurde, je älter die Patienten waren, je mehr sie unter einer somatischen Komorbidität zu leiden hatten und je seltener bei ihnen die Diagnosen Schizophrenie oder bipolare Erkrankung vorlagen. Auch dort haben die Kolleginnen und Kollegen das gerontopsychiatrische Kerngeschäft offensichtlich noch wenig ins Herz geschlossen. Es wird zu analysieren sein, ob und in welchem Umfang sich andere Fächer tatsächlich psychiatrischer Patienten annehmen, wie es eine Analyse von Versichertendaten [9] vor Einführung des DRG-Systems nahe legte. Die unterschiedliche Systematik der Finanzierung, bei der jetzt Fallpauschalen in allen somatischen Fächern den tagesgleichen Pflegesätzen in der Psychiatrie gegenüberstehen, macht dies für den stationären Bereich bis auf wenige Ausnahmen unwahrscheinlich. Bei ambulanten Patienten dagegen gibt es Hinweise, dass Nachbarfächer wie Neurologie oder Geriatrie durchaus Kompetenz und Zuständigkeit für psychiatrische Krankheitsbilder reklamieren.

Angesichts des Rückgangs der Beitragszahler bei steigendem finanziellen Bedarf für zusätzliche medizinische Technologien nimmt der Kostendruck auf das Gesundheitssystem unbezweifelbar zu. Die Frage nach der Allokation der vorhandenen Ressourcen, also nach der Verteilung knapper Güter auf konkurrierende Verwendungsweisen, ist aufgeworfen. Es konkurrieren aber nicht nur die Autobahn mit der Universität, das Schwimmbad mit der Schule oder das Museum mit dem Krankenhaus um öffentliche Mittel, es konkurrieren auch stationärer und ambulanter Bereich. Selbst auf der Ebene der Krankheiten findet ein Wettbewerb um knappe Ressourcen und um die öffentliche Gunst [4] statt.

Es gibt gute Gründe für die Annahme, dass zukünftig auch bei uns nicht mehr alle medizinisch angezeigten Maßnahmen für alle finanziert werden können, dass also eine Rationierung medizinischer Leistungen ins Auge gefasst wird, wie immer der politisch korrekte Euphemismus auch lauten mag. Das kalendarische Alter wird als Kriterium für die Rationierung medizinischer Güter und Dienstleistungen immer wieder angeführt. Aus der Vielzahl der in diesem Zusammenhang genannten Gründe [10] seien einige aufgeführt (nach: [11]): Alter sei ein einfach festzustellendes Kriterium, bei alten Patienten finde sich ein besonders hohes Einsparpotenzial, jüngere Menschen sollen eine höhere volkswirtschaftliche Produktivität aufweisen und schließlich sei das Alter als Rationierungskriterium nicht diskriminierend, da jeder früher oder später von den Vor- und Nachteilen betroffen sei.

Wie sieht es mit der Belastbarkeit dieser Argumente aus? Das Geburtsdatum ist sicher ein hartes Faktum, das allerdings nicht zur Identifizierung einer homogenen Gruppe der „Alten“ dient. Im Gegenteil findet sich eine mit dem Alter in den verschiedensten Bereichen zunehmende Differenzierung, sodass 30-Jährige einander wesentlich ähnlicher sind, als 70-Jährige. Entgegen einer auch in der Öffentlichkeit häufig zu vernehmenden Position sind die mit einer altersbezogenen Zuteilung medizinischer
Leistung verknüpften Einsparpotenziale nicht offensichtlich. Verschiedene Ergebnisse hinsichtlich der altersbezogenen Kostenverteilung belegen, dass die Krankenhauskosten für Hochbetagte deutlich niedriger liegen als die für jüngere Altersgruppen.

Nach einer Untersuchung von Brockmann [12] machten zwar die medizinischen Kosten im letzten Lebensjahr einen Großteil der gesamten Krankheitskosten aus, diese Kosten waren aber bei älteren Verstorbenen geringer als bei jüngeren. Man kann diese Daten auch als einen Hinweis auf eine bereits aktuell stattfindende subliminale Rationierung deuten. Die Argumente, die sich auf die höhere volkswirtschaftliche Produktivität junger Menschen und den höheren Gewinn an gesunden Lebensjahren bei ihnen beziehen, bedürfen als primär ökonomische Parameter zunächst einer ethischen Bewertung durch die Gesellschaft. Das letzte Argument behauptet eine Chancengleichheit, die schon jetzt nur als theoretisch anzusehen ist [11]. Es ist allerdings tröstlich zu wissen, dass die Orientierung am Gesamtzustand des Patienten selbst unter den Befürwortern des Alterskriteriums ein recht stabiles Regulativ darzustellen scheint [13].

Im Vierten Altenbericht der Bundesregierung [14] wird unterstrichen, dass alle Lebensphasen ethisch gleichrangig sind, was eine Abwertung des Alters und der Hochaltrigkeit ausschließt. Wenn also eine Begrenzung der Leistungen im Gesundheitswesen ansteht, so sollten deren Konsequenzen auf alle Altersgruppen gleichgewichtig verteilt werden. Die psychiatriespezifische Seite der Allokationsdebatte ist bisher wesentlich im Kontext der Antistigmadiskussion thematisiert worden. Die erwähnte Untersuchung von Schomerus et al. [4] fügt dem einen gerontopsychiatrischen Aspekt hinzu. Bei Telefoninterviews stellte sich heraus, dass die deutsche Bevölkerung Rationierungen von Gesundheitskosten eher bei psychiatrischen als bei somatischen Erkrankungen akzeptiert, wobei die Alkoholkrankheit besonders wenig öffentliche Akzeptanz genießt. Bemerkenswert ist aber, dass die Alzheimerkrankheit bei der Frage, welche Krankheiten bei einer möglicherweise notwendig werdenden Rationierung ausgenommen werden sollten, als einzige psychiatrische Erkrankung besser abschneidet als Rheumatismus und Diabetes und nur wenig schlechter als AIDS. Die öffentliche Akzeptanz der Alzheimerkrankheit könnte also auch für die Antistigmakampagne unseres Fachs Bedeutung gewinnen und es leichter machen, sich zur Gerontopsychiatrie zu bekennen.

\section{Literatur}

1 Stoppe G, Kipp J. Pro und Kontra: Separierte Versorgung gerontopsychiatrischer Patienten. Psychiat Prax 2006; 33: 258-260

2 Jeste DV, Alexopoulos GS, Bartels SJ, Cummings JL, Gallo JJ, Gottlieb GL, Halpain MC, Palmer BW, Patterson TL, Reynolds CF 3rd, Lebowitz BD. Consensus statement on the upcoming crisis in geriatric mental health: research agenda for the next 2 decades. Arch Gen Psychiatry 1999; 56: $848-853$

3 Gluck J, Baltes PB. Using the concept of wisdom to enhance the expression of wisdom knowledge: not the philosopher's dream but differential effects of developmental preparedness. Psychol Aging 2006; 21: 679-690

4 Schomerus G, Matschinger H, Angermeyer MC. Preferences of the public regarding cutbacks in expenditure for patient care. Soc Psychiatry Psychiatr Epidemiol 2006; 40: 1-9

5 Reister M. Diagnosedaten der Krankenhauspatienten 1994-1997. In: Arnold M, Litsch M, Schwartz FW (Hrsg): Krankenhaus-Report '99. Stuttgart: Schattauer, 2000: 273-288

6 Gutzmann H. Psychisch kranke alte Menschen: Wo sind sie geblieben? Spektrum 2001; 30: $31-33$

7 Bundesarbeitsgemeinschaft der Träger psychiatrischer Krankenhäuser (BAG Psychiatrie). Bericht über den Stand der klinisch-gerontopsychi- 
atrischen Versorgung in der Bundesrepublik Deutschland. Köln: BAG, 1997

8 Ettner SL. The setting of psychiatric care for medicare recipients in general hospitals with specialty units. Psychiatr Serv 2001; 52: 237 239

9 Maylath E, Spanka M, Nehr R. In welchen Krankenhausabteilungen werden psychisch Kranke behandelt? Eine Analyse der Krankenhausfälle der DAK im Vorfeld der DRGs. Gesundheitswesen 2003; 65: 486- 494

10 Buchner F, Hessel F, GreßS, Wasem J. Gesundheitsökonomische Aspekte des hohen Alters und der demographischen Entwicklung. In: Deutsches Zentrum für Altersfragen (Hrsg): Expertisen zum 4. Altenbericht der Bundesregierung, Band II, Ökonomische Perspektiven auf das hohe Alter. Hannover: Vincentz, 2002: 209-287
11 Helmchen H, Kanowski S, Lauter H. Ethik in der Altersmedizin. Stuttgart: Kohlhammer, 2006

12 Brockmann $H$. Why is less money spent on health care for the elderly than for the rest of the population? Health care rationing in German hospitals. Soc Sci Med 2002; 55: 593-608

13 Callahan D. Setting limits. Medical goals in an aging society. New York: Simon \& Schuster, 1987

14 Bundesministerium für Familie, Senioren, Frauen und Jugend (BMFSFJ). Vierter Bericht zur Lage der älteren Generation in der Bundesrepublik Deutschland: Risiken, Lebensqualität und Versorgung Hochaltriger unter besonderer Berücksichtigung demenzieller Erkrankungen. Berlin: BMFSFJ, 2002 